\title{
Lattice Measurement of the Delta $I=1 / 2$ Contribution to Standard Model Direct CP-Violation in $K \rightarrow \pi \pi$ Decays at Physical Kinematics: Part II
}

\author{
D.Zhang* \\ Columbia University \\ E-mail: dz2203ecolumbia.edu \\ C.Kelly \\ RIKEN-BNL Research Center, \\ E-mail: ckelly@quark.phy.bnl.gov
}

\begin{abstract}
In continuation of part I, I will discuss our use of all-to-all propagators to construct pi-pi operators with reduced coupling to the vacuum, which helps to drcrease the noise in the $K \rightarrow \pi \pi(I=0)$ decay amplitude. I will also present preliminary results for the $\pi \pi(I=0)$ phase shift and the $I=0, K \rightarrow \pi \pi$ lattice matrix elements, both with physical kinematics. The precise value of $A_{0}$, once computed, will determine the measure of the direct CP-violation $\varepsilon^{\prime}$ from the Standard Model.
\end{abstract}

The 32nd International Symposium on Lattice Field Theory,

23-28 June, 2014

Columbia University New York, NY

${ }^{*}$ Speaker. 


\section{Motivations}

In the Standard Model, the measure of direct CP-violation in $K \rightarrow \pi \pi$ decay is determined by:

$$
\varepsilon^{\prime}=\frac{i e^{i\left(\delta_{2}-\delta_{0}\right)}}{\sqrt{2}} \frac{\operatorname{Re} A_{2}}{\operatorname{Re} A_{0}}\left(\frac{\operatorname{Im} A_{2}}{\operatorname{Re} A_{2}}-\frac{\operatorname{Im} A_{0}}{\operatorname{Re} A_{0}}\right),
$$

where $\delta_{2}$ and $\delta_{0}$ are the strong phase shifts from $\pi \pi$ scattering, $A_{2}$ and $A_{0}$ are the two decay amplitudes corresponding to the two different final $\pi \pi$ isospin states. Using lattice QCD, $A_{2}$ is now calculated with physical kinematics and physical quark mass[1], with error bars less than $10 \%$. To calculate $\varepsilon^{\prime}$, the only remaining unknown quantity is $A_{0}$.

In the experimental measurement of $K \rightarrow \pi \pi$ decay, the evidence of direct $\mathrm{CP}$ violation comes from the measurement $\left(1-\left|\eta_{00} / \eta_{+-}\right|\right) / 3=\operatorname{Re}\left(\varepsilon^{\prime} / \varepsilon\right)=(1.65 \pm 0.26) 10^{-3}[2]$, where the two $\eta$ quantities are defined as

$$
\begin{gathered}
\eta_{+-}=\frac{A\left(K_{L} \rightarrow \pi^{+} \pi^{-}\right)}{A\left(K_{S} \rightarrow \pi^{+} \pi^{-}\right)} \\
\eta_{00}=\frac{A\left(K_{L} \rightarrow \pi^{0} \pi^{0}\right)}{A\left(K_{S} \rightarrow \pi^{0} \pi^{0}\right)} .
\end{gathered}
$$

The experimental error bar for $\operatorname{Re}\left(\varepsilon^{\prime} / \varepsilon\right)$ is about $16 \%$. In order to compare that with the Standard Model prediction of $\varepsilon^{\prime}$, a precise lattice calculation of $A_{0}$ is needed.

The following sections will describe our strategies to calculate the physical decay amplitude $A_{0}$ as well as the current status of our calculation.

\section{Measurement Strategy}

Compared to previous lattice measurement, we use two new strategies: 1. G-parity boundary condition, in order to give physical kinematics to the final two-pion state; 2. All-to-all propagators, in order to control the vacuum fluctuation in disconnected diagrams.

\subsection{G-parity boundary condition}

G-parity boundary conditions are only used in the spatial directions. The G-parity conventions we use are supposing a G-parity boundary in $\hat{z}$ direction):

$$
\begin{aligned}
& u_{x+L \hat{z}}=\left(\bar{d}_{x} \gamma_{4} \gamma_{2}\right)^{T}=-\gamma_{4} \gamma_{2} \bar{d}_{x}^{T} \\
& \bar{u}_{x+L \hat{z}}=\left(\gamma_{4} \gamma_{2} d_{x}\right)^{T}=-d_{x}^{T} \gamma_{4} \gamma_{2} \\
& d_{x+L \hat{z}}=-\left(\bar{u}_{x} \gamma_{4} \gamma_{2}\right)^{T}=\gamma_{4} \gamma_{2}{\overline{u_{x}}}^{T} \\
& \bar{d}_{x+L \hat{z}}=-\left(\bar{d}_{x} \gamma_{4} \gamma_{2}\right)^{T}=u_{x}^{T} \gamma_{4} \gamma_{2} .
\end{aligned}
$$

It can be shown that under this definition, $\mathrm{SU}(2)$ isospin symmetry is preserved and each pion satisfies anti-periodic boundary conditions:

$$
\begin{aligned}
& \pi_{x+L \hat{z}}^{+}=-\pi_{x}^{+} \\
& \pi_{x+L \hat{z}}^{0}=-\pi_{x}^{0} \\
& \pi_{x+L \hat{z}}^{-}=-\pi_{x}^{-} .
\end{aligned}
$$


Under these boundary conditions, the single pion ground state now carries one half the lattice momentum $\frac{2 \pi}{L}$. By carefully tuning the lattice size, we can give physical kinematics for the $K \rightarrow \pi \pi$ decay.

One by-product of G-parity boundary conditions is extra Wick contractions. As a simple example, the pion correlation function now looks like:

$$
\langle\pi \pi\rangle=\overline{\bar{u} \gamma_{5} d \quad \bar{d} \gamma_{5} u}+\overline{\bar{u} \gamma_{5} d \quad \bar{d} \gamma_{5} u},
$$

where the first term is the usual contraction term, while the second term arises because the quark lines could cross the G-parity boundary. Thus a $d$ quark could change flavor and contract with a $u$ quark as shown in Figure (1). This extra contraction will not change the SU(2) iso-spin quantum number since $u$ and $d$ have the opposite iso-spin. Because the G-parity boundary mixes the two quark flavors, the number of possible Wick contractions grows rapidly as the correlation function becomes more complicated. For example, the $\left\langle\pi \pi\left|H_{w}\right| K\right\rangle$ matrix element could contain as many as 256 different contractions.

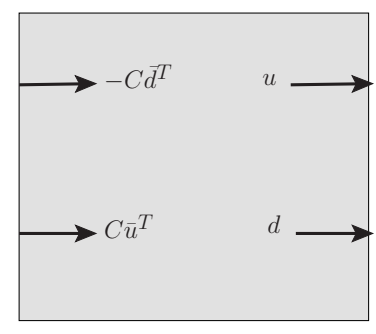

Figure 1: G-parity boundary mixes the two quark flavors.

G-parity boundary conditions also put constraints on the quarks' momenta. G-parity boundary means that quarks will satisfy anti-periodic boundary conditions in a double-sized lattice, since

$$
u(x+2 L)=-\gamma_{4} \gamma_{2} \bar{d}^{T}(x+L)=-u(x) .
$$

This means quarks can only have momentum components of the form $\left(n+\frac{1}{2}\right) \frac{\pi}{L}$, with $\mathrm{n}$ being an integer, in each of the spatial directions with G-parity boundary conditions. Besides that constraint, there is also constraint on the momentum direction. This can be seen by writing down a momentum eigenstate of the quark field. A momentum eigenstate is defined by:

$$
T_{z} \widetilde{\psi}\left(p_{z}\right) T_{z}^{-1}=e^{i p_{z}} \widetilde{\psi}\left(p_{z}\right),
$$

where the two-component $\psi$ field is composed of the two SU(2) flavors: $\psi=\left(\begin{array}{c}\psi^{(0)} \\ \psi^{(1)}\end{array}\right)=\left(\begin{array}{c}d \\ C \bar{u}^{T}\end{array}\right)$ and transforms when passing through the G-parity boundary as:

$$
\begin{aligned}
& \psi\left(L_{z}\right)=i \sigma_{2} \psi(0) \\
& \bar{\psi}\left(L_{z}\right)=-i \bar{\psi}(0) \sigma_{2},
\end{aligned}
$$

and the translation operator $T_{z}$ shifts the field operator by one unit:

$$
T_{z} \psi(z) T_{z}^{-1}=\psi(z-1) .
$$


The momentum eigenstate of the quark field is then:

$$
\begin{aligned}
\widetilde{\psi}\left(p_{z}\right) & =\sum_{n_{z}=0}^{L_{z}-1}\left\{e^{-i p_{z} n_{z}} \psi\left(n_{z}\right)+i \sigma_{2} e^{-i p_{z}\left(n_{z}+L_{z}\right)} \psi\left(n_{z}\right)\right\} \\
& =\left(1+i e^{-i p_{z} L_{z}} \sigma_{2}\right) \sum_{n_{z}=0}^{L_{z}-1}\left\{e^{-i p_{z} n_{z}} \psi\left(n_{z}\right)\right\} .
\end{aligned}
$$

Translating in z-direction by one unit, the two components at (and right before) the G-parity boundary in the bracket of equation (2.14) will transform into each other, leaving an overall phase factor as in equation (2.10). Using $p_{z}=\left(n_{z}+\frac{1}{2}\right) \frac{\pi}{L}$, equation (2.14) becomes

$$
\widetilde{\psi}\left(p_{z}\right)=\left(1+(-1)^{n_{z}} \sigma_{2}\right) \sum_{n_{z}=0}^{L_{z}-1}\left\{e^{-i p_{z} n_{z}} \psi\left(n_{z}\right)\right\}
$$

With three G-parity twists, the prefactor in equation (2.16) changes from $\left(1+(-1)^{n_{z}} \sigma_{2}\right)$ to $(1+$ $\left.(-1)^{n_{x}} \sigma_{2}\right)\left(1+(-1)^{n_{y}} \sigma_{2}\right)\left(1+(-1)^{n_{z}} \sigma_{2}\right)$. Since $\left(1-\sigma_{2}\right)$ is orthogonal to $\left(1+\sigma_{2}\right)$, all the $n_{x}, n_{y}, n_{z}$ must have the same parity, otherwise $\widetilde{\psi}\left(p_{z}\right)$ vanishes. Figure (2) shows the constraint that the G-parity boundary puts on the quark momentum.

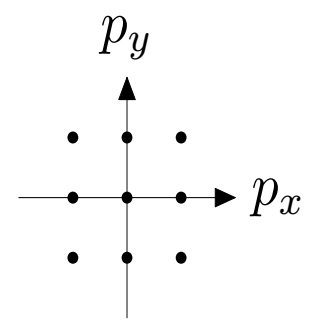

No G-parity twist

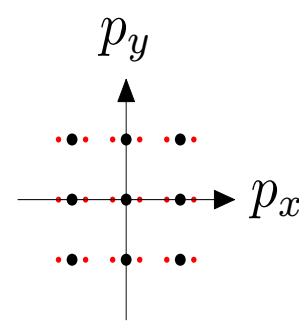

1 G-parity twist

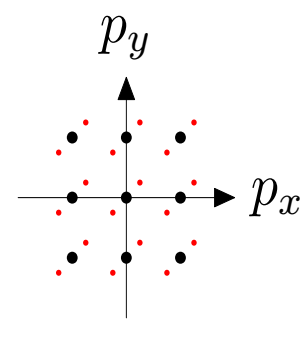

2 G-parity twists

Figure 2: Large black dots: allowed quark momentums with periodic boundary conditions; small red dots: allowed quark momentums with G-parity boundary conditions.

A consequence of this momentum constraint is that the cubic symmetry of the fermion action is broken. Fortunately this shouldn't affect the cubic symmetry of pion operator much. Even though the quarks are required to carry momenta only in the 'diagonal' direction as shown in Figure (2), the pion's momentum is not constrained in this way and G-parity boundary conditions are the same as anti-periodic boundary conditions for the pion. However the cubic symmetry breaking at the fermion action level affects the pion operator in a more subtle way: the difference between pion operators with diagonal momentum and off-diagonal momentum lies in the quarks' momenta in each pion: the pion with momentum $\vec{p}_{\pi}=\left(\frac{1}{2}, \frac{1}{2}, \frac{1}{2}\right)$ can have two quarks each carrying the same momentum of $\vec{p}_{\text {quark }}=\left(\frac{1}{4}, \frac{1}{4}, \frac{1}{4}\right)$; but the pion with momentum $\vec{p}_{\pi}=\left(\frac{-1}{2}, \frac{1}{2}, \frac{1}{2}\right)$ can't have two quarks each carrying the same momentum of $\vec{p}_{\text {quark }}=\left(\frac{-1}{4}, \frac{1}{4}, \frac{1}{4}\right)$, for the similar reason that the red points in "2 G-parity twists" graph in Figure (2) only lie in the diagonal direction. As a result, the pion with momentum $\vec{p}_{\pi}=\left(\frac{-1}{2}, \frac{1}{2}, \frac{1}{2}\right)$ must have nonzero relative momentum between its two quark components.

This difference between pion momentum eigenstates is caused by the G-parity boundary conditions and should be exponentially suppressed when the lattice volume becomes large, due to 
the short distance nature of the strong interaction. On the $32^{3} \times 64$ ensemble that we are using, this rotation symmetry breaking effect can't be detected, by comparing norms of pion momentum eigenstates as in Table (1).

\begin{tabular}{ccccc}
\hline & $\mathrm{p}=(+,+,+)$ & $\mathrm{p}=(-,+,+)$ & $\mathrm{p}=(+,-,+)$ & $\mathrm{p}=(+,+,-)$ \\
\hline$E_{\pi}$ & $0.19852(85)$ & $0.19823(82)$ & $0.19839(72)$ & $0.19866(88)$ \\
$Z_{\pi}$ & $6.167(69) \mathrm{e}+06$ & $6.081(63) \mathrm{e}+06$ & $6.183(50) \mathrm{e}+06$ & $6.170(61) \mathrm{e}+06$ \\
\hline
\end{tabular}

Table 1: The pion energy and norm, with different momentum directions. ' + ' corresponds to $+3.14 / \mathrm{L}$ momentum, '-' corresponds to $-3.14 / \mathrm{L}$ momentum. Using 24 configuration.

\subsection{All-to-all propagators}

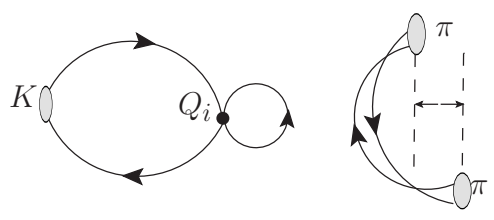

Figure 3: Disconnected diagram in $K \rightarrow \pi \pi$ using all-to-all propagators. The two pions are separated in time direction as another technique to reduce the vacuum fluctuation.[3]

One advantage of using all-to-all propagators is that it reduces noise. Since the $\pi \pi(I=0)$ state is involved in the $I=0, K \rightarrow \pi \pi$ decay process, disconnected diagrams occur. This is shown in Figure (3), the $\pi \pi(I=0)$ operator could connect to itself, and no quark line goes between the weak operator and the $\pi \pi(I=0)$ operator. As a result, the amplitude of the disconnected diagram falls off slowly as the time separation increases, while the connected diagrams fall off much faster. So even small fluctuations in the disconnected diagrams could deteriorate the overall signal a lot. Allto-all propagators reduce the fluctuation in the disconnected diagrams, by reducing the coupling between $\pi \pi(I=0)$ state and the vacuum[4].

The all-to-all propagator method also saves time in computing quark propagators with various momentum directions. When using the traditional method to calculate a momentum wall source quark propagator, we need one solve for each quark momentum:

$$
G\left(x^{\prime}, t_{2} ; p, t_{1}\right)=\sum_{x} D^{-1}\left(x^{\prime}, t_{2} ; x, t_{1}\right) e^{-i p x},
$$

where $p$ is the quark momentum. In the presence of G-parity boundary in 3 spatial directions, the allowed quark momenta are $p=\left(n_{1}, n_{2}, n_{3}\right) \frac{2 \pi}{L} \pm(1,1,1) \frac{\pi}{2 L}$ with $n_{i}$ being integers, and it turns out that we need at least 4 of them to construct a two-pion operator which has the greatest degree of cubic symmetry. In our current $K \rightarrow \pi \pi(I=0)$ measurement job, it takes $30 \%$ of the total time to compute the light quark propagator with only one momentum (on all 64 time slices). It's obvious that using all-to-all propagators saves a significant amount of computational time.

In contrast, an all-to-all propagator does not need any extra solves for multiple quark momenta, because it already contains the propagation information from an arbitrary source point to an arbitrary sink point, up to random noise[4]:

$$
G\left(x^{\prime}, t_{2} ; x, t_{1}\right) \approx \sum_{i} v^{i}\left(x^{\prime}, t_{2}\right) w^{i \dagger}\left(x, t_{1}\right),
$$


and the quark momentum are included by doing a Fourier transform:

$$
G\left(x^{\prime}, t_{2} ; p, t_{1}\right) \approx \sum_{x} \sum_{i} v^{i}\left(x^{\prime}, t_{2}\right) w^{i^{\dagger}}\left(x, t_{1}\right) e^{-i p x} .
$$

Different quark momenta amount to different Fourier transforms, and no extra inversion of the Dirac operator is needed.

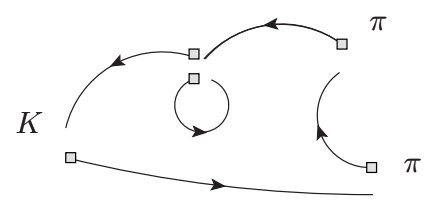

Figure 4: Type3 diagram in $K \rightarrow \pi \pi$, using all-to-all propagators

Though all-to-all propagators reduce fluctuation in disconnected diagrams and save inversion time, they make the contractions expensive. As shown in Equation (2.18), when using all-to-all propagators to evaluate the Green's function, the time complexity is a linear function of the number of modes. When evaluating a more complex diagram like in Figure (4), there are four quark lines converging at the weak Hamiltonian operator, which requires the summation of four mode indices (each quark line brings one mode index) and is not trivial to do. The algorithm we use to evaluate the diagrams in $K \rightarrow \pi \pi(I=0)$ has a time complexity of $O\left(V * N^{2}\right)$, where $V$ is the 4D volume and $N$ is the number of modes.

\section{Current result}

\begin{tabular}{cccccc}
\hline & $m_{K}$ & $E_{\pi}$ & $E_{\pi \pi(I=2)}$ & $E_{\pi \pi(I=0 V)}$ & $E_{\pi \pi(I=0)}$ \\
\hline lattice & $0.35539(23)$ & $0.19898(42)$ & $0.41435(80)$ & $0.3616(50)$ & $0.3805(327)$ \\
\hline $\mathrm{MeV}$ & $490.44 \pm 0.32$ & $274.59 \pm 59$ & $571.8 \pm 1.1$ & $499.0 \pm 6.9$ & $525 \pm 45$ \\
\hline
\end{tabular}

Table 2: Meson spectrum in both lattice units and MeV. Measured on 87 configurations. The $E_{\pi \pi(I=0 V)}$ column is the $\pi \pi(I=0)$ energy without including vacuum diagrams.

By measuring 87 configurations spaced by 8 molecular-dynamic time units, we obtained the meson spectrum as in Table (2). Using the $E_{\pi \pi}$ value in Table (2) and Luscher's quantization condition [5], we can calculate the strong phase shifts:

$$
\begin{aligned}
& \delta_{2}=-0.1939(87)(\text { radians }) \\
& \delta_{0}=0.18(36)(\text { radians })
\end{aligned}
$$

The ten weak matrix elements on lattice $M_{i}^{\text {lat }}=\left\langle\pi \pi(I=0)\left|Q_{i}\right| K\right\rangle(i=1,2, \cdots, 10)$ are also calculated, each with the two quark, dimension 3 operator $\bar{s} \gamma_{5} d$ subtraction:

$$
Q_{i}^{s u b}=Q_{i}-\alpha_{i} \bar{s} \gamma_{5} d
$$

with $\alpha$ chosen so that

$$
\left\langle 0\left|Q_{i}^{s u b}\right| K\right\rangle=0
$$


Because of the relative sizes of the matrix elements $M_{i}^{\text {lat }}(i=1,2, \cdots, 10)$ and the perturbative Wilson coefficients, $\operatorname{Re}\left(A_{0}\right)$ is mostly determined by the second matrix element $M_{2}^{\text {lat }}$, while $\operatorname{Im}\left(A_{0}\right)$ is mostly determined by the sixth matrix element $M_{6}^{\text {lat }}$. We obtained the lattice matrix elements at various $K-\pi \pi$ separations as shown in Table (3).

\begin{tabular}{|c|c|c|}
\hline$\delta t$ & $M_{2}^{\text {lat }}\left(\times 10^{-3}\right)$ & $M_{6}^{\text {lat }}\left(\times 10^{-3}\right)$ \\
\hline 10 & $3.1(43)$ & $-25(11)$ \\
12 & $5.2(30)$ & $-27(10)$ \\
14 & $1.8(32)$ & $-16(10)$ \\
\hline Average & $3.4(24)$ & $-23.8(74)$ \\
\hline
\end{tabular}

Table 3: Matrix elements $M_{2}$ and $M_{6}$. They were obtained from 87 configurations, substantial more data than was available at the time of presentation during the symposium.

By a rough estimation, we need to measure on 300 configurations in order to get the $A_{0}$ with less than $30 \%$ uncertainty.

\section{Acknowledgement}

This work is supported by the U.S. Department of Energy (DOE) under grant DE-SC0011941. The BG/Q resources are provided by the RIKEN BNL Research Center, the DOE funded USQCD machine at Brookhaven National Laboratory, and the Mira computer in the ALCF at Argonne National Laboratory. I will also thank my advisor Norman H. Christ and all other RBC\&UKQCD colleagues for important suggestions and useful discussions.

\section{References}

[1] T. Blum, P.A. Boyle, N.H. Christ, N. Garron, E. Goode, et al. Lattice determination of the $K \rightarrow(\pi \pi)_{I=2}$ Decay Amplitude $A_{2}$. Phys.Rev., D86:074513, 2012.

[2] K. Nakamura et al. Review of particle physics. J.Phys., G37:075021, 2010.

[3] Qi Liu. Kaon to two pions decays from lattice $Q C D: \Delta I=1 / 2$ rule and $C P$ violation. $\mathrm{PhD}$ thesis, Columbia University, 2012.

[4] D. Zhang. Using all-to-all propagators for $K \rightarrow \pi \pi$ decays. PoS, LATTICE2013:403, 2013.

[5] Martin Luscher. Two particle states on a torus and their relation to the scattering matrix. Nucl.Phys., B354:531-578, 1991. 